\title{
Ewing's Sarcoma of the Sternum: A Case Report and Literature Review
}

\author{
Ahmed Dehal ${ }^{1 *}$, Hannah Copeland ${ }^{2}$, Albert Kheradpour ${ }^{2}$, Mark Martin $^{2}$, Jason Wallen ${ }^{2}$ and Salman Zaheer ${ }^{2}$ \\ ${ }^{1}$ Kaiser Permanente, Fontana, CA, USA \\ 2Loma Linda University Medical Center, Loma Linda, CA, USA \\ "Corresponding author: Ahmed Dehal, MD, MPH, Department of General Surgery, Kaiser ermanente, Fontana, CA, USA, Tel: (909)247-5626; Fax: (909)247-4626; E- \\ mail: ahmed.dehal@gmail.com
}

Received Date: 28 February 2014, Accepted Date: 20 March 2014, Published Date: 30 March 2014

Copyright: (c) 2014 Dehal A, et al. This is an open-access article distributed under the terms of the Creative Commons Attribution License, which permits unrestricted use, distribution, and reproduction in any medium, provided the original author(s) and source are credited.

\begin{abstract}
A 16 year old male presented with Ewing's sarcoma of the sternum which extended into the anterior mediastinum. At presentation, there was no evidence of metastatic disease. The patient was initially treated with chemotherapy. The residual tumor was subsequently resected and the sternal defect was repaired with a polyprolene mesh and a muscle flap. Post-operatively, the patient did well and was discharged home. The patient has been seen in the clinic and is doing well post-operatively. Although extremely rare, Ewing's sarcoma occurs in the sternum. A combined effort between oncology, thoracic surgery and plastic surgery for single excision and reconstruction is optimal for the patient.
\end{abstract}

Keywords: Ewing; Sarcoma; Sternum; Case; Report

\section{Introduction}

Ewing sarcoma is a solid tumor of bone and soft tissue can arise in any bone of the body. However, the most common sites of disease include the pelvis, ribs, and long-bones of the extremities. Ewing's sarcoma of the sternum has been rarely reported in the literature. Most neoplasms of the sternum are metastases. Primary tumors are relatively uncommon in this site; however, primary tumors of the sternum are much more frequently malignant than benign. Whether it is believed to be primary or secondary, a new mass in the sternum should be considered malignant until proven otherwise [1].

These tumors are most often found in children (median age, 16 years). They manifest clinically as painful masses. Metastases to lung, bone, and lymph nodes are seen in approximately $70 \%$ of patients with Ewing sarcoma. Overall 5-year and 10-year survival is only approximately $50 \%$, and the outcome is ultimately determined by the presence of metastasis. Survival after resection is also dependent on the histology and grade of the tumor [2].

Since the tumor was first described more than half a century ago, the subject has been marked by controversy and uncertainty, and approaches to therapy have varied widely. The treatment of Ewing sarcoma relies on a multidisciplinary approach, coupling highly intensive chemotherapy with surgery, and/or radiotherapy. We report a case of a Ewing's sarcoma of the sternum that was treated with chemotherapy and surgery.

\section{Case Report}

A 16 year old male had an enlarging chest wall mass, tenderness and a $30 \mathrm{lb}$ weight loss. The patient denied any chest pain or shortness of breath. The child was an otherwise healthy with no history of other medical problems. Computed Tomography (CT) scan of the chest showed anterior sternum calcified mass extending into the anterior mediastinum (Figure 1). The mass was biopsied and found to be Ewing's sarcoma. The patient was treated with 12 cycles of chemotherapy (vincristine, cyclophosamide, and topotecan). The tumor decreased in size by two-thirds its original size. Five months later the tumor was excised in a circumferential fashion with $1.5 \mathrm{~cm}$ negative margins (Figure 2). One third of the sternum was removed along with adjacent intercostal muscles and rib ends. The plastic surgery team placed alloderm over the heart, polyprolene mesh, mobilized a left lattisimus dorsi muscle flap and tunneled it under the left chest over the sternal space. Three sternal plates were placed across the sternum. Post-operatively, the patient did well and was discharged to home on post-operative day 10 . The patient has been seen in the clinic and is doing well post-operatively (Figure 3 ). He is now one year after diagnosis and he is in good health with no evidence of recurrent tumor.

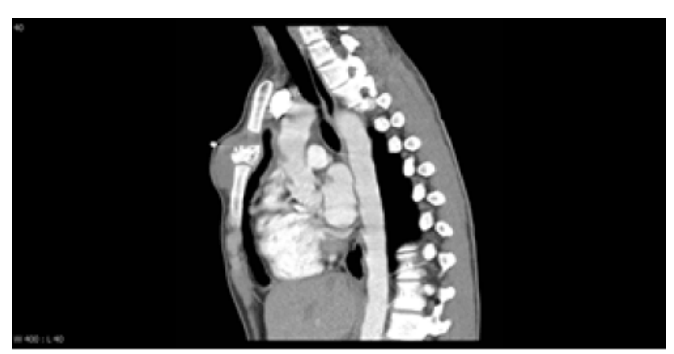

Figure 1: CT scan of the chest showing the sarcoma of the sternum.

\section{Discussion}

Primary Ewing's sarcoma of the sternum is extremely rare. Only a few studies have reported on Ewing's sarcoma of the sternum, and nearly all of them had small patient populations. A literature search revealed only four reported cases of primary Ewing's sarcoma of the sternum [3-6]. However, no clinical data were available for three of those cases. Ewing's sarcoma of the sternum has not been recorded in the Intergroup Ewing's Sarcoma Study [7]. It was also not noted in 
several old series ranging from 12 to 73 cases (totaling 537 cases) of this cancer [8-17].

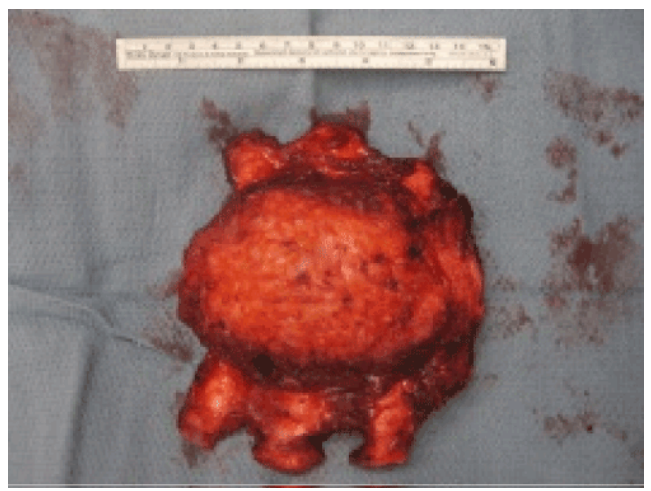

Figure 2: Resected sarcoma specimen.

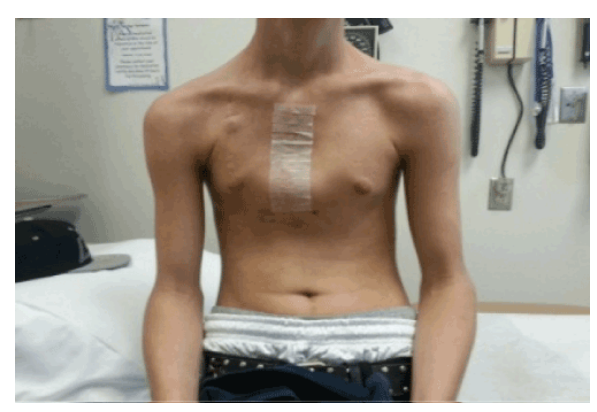

Figure 3: Postoperative wound.

The first case was reported in a 12 years old female who was successfully treated with chemotherapy and radiation followed by surgery [3]. The second case was found in a series of 107 cases from Tara Memorial Hospital in Bombay, India [4]. The third case was reported in a large study of 11,087 bone tumors in the Dahlin tumor series at the Mayo Clinic. In their study, only $66(0.6 \%)$ were primary malignant tumors of the sternum. Among those, only 66 were primary sternal malignancies including 22 chondrosarcomas (33\%), 20 myelomas (including plasmacytomas) (30\%), 14 lymphomas (21\%), eight osteosarcomas (12\%), one fibrosarcoma (1.5\%), and one Ewing sarcoma (1.5\%) [5]. The last case was reported in the most recent report from Memorial Sloan Kettering Cancer Center describing their experience with primary and secondary malignancies of the sternum over 69-year period (1930 to 1999). In their series of 58 patients with primary sternal malignancies were identified. Among this, there were 28 chondrosarcomas (48\%), 11 osteosarcomas (19\%), nine plasmacytomas (15.5\%), six lymphomas (10\%), one fibrosarcoma (1.7\%), one angiosarcoma (1.7\%), one malignant fibrous histiocytoma (1.7\%), and one Ewing sarcoma (1.7\%), [6].

Tumors of the sternum have been considered a challenging problem for a long time. Stability and reconstruction of chest wall defects have caused the main difficulties in radical full-thickness resections. Improvement of surgical techniques, especially by means of myocutaneous flaps and prosthetic materials, has resulted in successful sternectomies and simultaneous reconstructions. Incarbone et al in their series of 52 patients who underwent sternal resection for primary and secondary tumor concluded that extensive resection of the sternum for primary or secondary tumors followed by reconstruction with prosthetic material or a myocutaneous flap is a safe and effective treatment [18].

Multimodal approaches within clinical trials, employing combination chemotherapy and surgery and/or radiotherapy, have raised 5-year survival rates from $<10 \%$ to $>60 \%$. The current recommendations by the European Society for Medical Oncology Group consider complete surgery, where feasible, as the best modality of local control. Radiotherapy should be applied only if complete surgery is impossible, and should be discussed where histological response in the surgical specimen is poor (i.e. $>10 \%$ viable tumor cells) [19].

All current trials employ three to six cycles of initial chemotherapy after biopsy, followed by local therapy and another six to ten cycles of chemotherapy usually applied at 3-week intervals. Treatment duration is thus 8-12 months. Agents considered most active include doxorubicin, cyclophosphamide, ifosfamide, vincristine, dactinomycin and etoposide. Virtually all active protocols are based on four- to sixdrug combinations of these substances. According to findings from the European Intergroup Cooperative Ewing's Sarcoma Study, protocols that have proved to be most effective include at least one alkylating agent (ifosfamide or cyclophosphamide) and doxorubicin [20].

In a large series of adult Ewing sarcoma patients from the Mayo Clinic, outcomes were analyzed for 102 patients. The authors reported improved results in the modern portion of their study (1993 through 2007 versus 1977 through 1992) reflecting a move toward using surgery along with etoposide and ifosfamide-based chemotherapy. The authors reported a five-year overall survival rate of $73 \%$ and a five-year event free survival rate of $60 \%$ in their modern group. Furthermore, local failure was $18 \%$ in the surgery group, compared with $33 \%$ in the radiation group and $0 \%$ with the combination of the two local modalities [21].

Tumor resection of Ewing's sarcoma in the sternum can be performed without mutilation. Our patient has only a mild cosmetic defect. Bony resection has a particular advantage in the sternum, where long-term surgical morbidity is small. Thus, neoadjuvant chemotherapy followed by excision in this site should certainly be considered. We presented a case of Ewing's sarcoma of the sternum in a 16 years old male who was successfully treated with chemotherapy and surgery. A combined effort between oncology, thoracic surgery and plastic surgery for single excision and reconstruction is optimal for the patient.

\section{Acknowledgement}

This manuscript has not been submitted for publication elsewhere. All the authors have contributed significantly, and all authors are in agreement with the content of the manuscript.

Conflict of Interest Statement: The authors indicate no potential conflict of interest or financial ties.

\section{References}

1. Downey RJ, Huvos AG, Martini N (1999) Primary and secondary malignancies of the sternum. Semin Thorac Cardiovasc Surg 11: 293-296.

2. Burt M (1994) Primary malignant tumors of the chest wall. The Memorial Sloan-Kettering Cancer Center experience. Chest Surg Clin N Am 4: 137-154. 
3. Kedar A, Ghoorah J, Thomas PR, Mindell ER, Tebbi CK, et al. (1979) Primary Ewing's sarcoma of the sternum: a case report. Med Pediatr Oncol 7: 163-167.

4. Bhansali SK, Desai PB (1963) Ewing's sarcoma: observations on 107 cases. J Bone Joint Surg Am 45: 541-553.

5. Unni KK (1996) Dahlin's bone tumors: general aspects and data on 11,087 cases. Philadelphia, Pa: Lippincott-Raven.

6. Downey RJ, Huvos AG, Martini N (1999) Primary and secondary malignancies of the sternum. Semin Thorac Cardiovasc Surg 11: 293-296.

7. Razek A, Perez CA, Tefft M, Nesbit M, Vietti T, et al. (1980) Intergroup Ewing's Sarcoma Study: local control related to radiation dose, volume, and site of primary lesion in Ewing's sarcoma. Cancer 46: 516-521.

8. Freeman AI, Sachatello C, Gaeta J, Shah NK, Wang JJ, et al. (1972) An analysis of Ewing's tumor in children at Roswell Park Memorial Institute. Cancer 29: 1563-1569.

9. Fernandez CH, Lindberg RD, Sutow WW, Samuels ML (1974) Localized Ewing's sarcoma--treatment and results. Cancer 34: 143-148.

10. Gasparini M, Barni S, Lattuada A, Musumeci R, Bonadonna G, et al (1977) Ten years' experience with Ewing's sarcoma. Tumori 63: 77-90.

11. Jenkin RD (1966) Ewing's sarcoma a study of treatment methods. Clin Radiol 17: 97-106.

12. Nouri MM, Hashemian H (1975) Ewing's tumor. Review of 73 cases. Int Surg 60: 478-481.

13. Palma J, Gailani S, Freeman A, Sinks L, Holland JF (1972) Treatment of metastatic Ewing's sarcoma with BCNU. Cancer 30: 909-913.

14. Rosen G, Wollner N, Tan C, Wu SJ, Hajdu SI, et al. (1974) Proceedings: Disease-free survival in children with Ewing's sarcoma treated with radiation therapy and adjuvant four-drug sequential chemotherapy. Cancer 33: 384-393.

15. Wang CC, Schulz MD (1953) Ewing's sarcoma; a study of fifty cases treated at the Massachusetts General Hospital, 1930-1952 inclusive. N Engl J Med 248: 571-576.

16. Walsh GL, Davis BM, Swisher SG, Vaporciyan AA, Smythe WR, et al. (2001) A single-institutional, multidisciplinary approach to primary sarcomas involving the chest wall requiring full-thickness resections. J Thorac Cardiovasc Surg 121: 48-60.

17. Hoeffel JC, Stines J, Schwab MP (1994) Les tumeurs primitives du sternum. Rev In Med 6:681-685.

18. Incarbone M, Nava M, Lequaglie C, Ravasi G, Pastorino U (1997) Sternal resection for primary or secondary tumors. J Thorac Cardiovasc Surg 114: 93-99.

19. Paulussen $M$, Bielack $S$, Jürgens $H$ on behalf of the ESMO Guidelines Working Group (2009) Ewing's sarcoma of the bone: ESMO Clinical Recommendations for diagnosis, treatment and follow-up. Annals of Oncology 140-142.

20. Paulussen M, Craft AW, Lewis I, Hackshaw A, Douglas C, et al. (2008) Results of the EICESS-92 Study: two randomized trials of Ewing's sarcoma treatment--cyclophosphamide compared with ifosfamide in standard-risk patients and assessment of benefit of etoposide added to standard treatment in high-risk patients. J Clin Oncol 26: 4385-4393.

21. Ahmed SK, Robinson SI, Okuno SH, Rose PS, Laack NN (2013) Adult Ewing sarcoma: survival and local control outcomes in 102 patients with localized disease. Sarcoma 68: 1425. 